\title{
Energy-Aware Relay Selection for Multiuser Relay Networks
}

\author{
Fangzhi Zuo and Min Dong \\ Faculty of Engineering and Applied Science, University of Ontario Institute of Technology, Canada \\ Email: \{fangzhi.zuo, min.dong\}@uoit.ca
}

\begin{abstract}
We consider a dual-hop relay network with multiple source-destination (S-D) pairs and multiple relays, where amplify-and-forward relaying strategy is applied and transmission among S-D pairs takes place simultaneously. Network lifetime in this scenario is defined as the time interval over which successful transmission of all S-D pairs through selected relays can be maintained. We aim at designing relay selection to maximize the network lifetime for given data rate requirements of all the S-D pairs. Without knowledge of future channel states, we design relay selection algorithms to maximize perceived network lifetime at the current time. The perceived network lifetime maximization is shown to be a max-min optimization problem. We propose a priority search algorithm which is shown to provide the optimal solution with linear complexity in the number of relays. Furthermore, we propose a suboptimal priority-based selection strategy, the "worst-case" greedy algorithm, with complexity linear in the number of relays and quadratic in the number of S-D pairs. Simulation results show that the performance loss of the "worst-case" greedy algorithm is negligible as compared to the optimal relay selection solution.
\end{abstract}

\section{INTRODUCTION}

Cooperative relaying architecture has been adopted for future-generation wireless systems for broadband access and coverage improvement. In some relaying applications, it is likely that relays are battery-powered, such as stand-alone relay stations away from the power grid, or peer users in an ad hoc network or multi-hop cellular network. Extending the lifetime of such networks becomes pivotal to maintain the quality of service with uninterrupted data exchange and to reduce the need for replenishing the batteries. There has been much research on energy-efficient packet forwarding in the ad hoc and sensor networking paradigms. However, studies on lifetime maximization for physical-layer cooperative relaying have so far been few, especially in multiuser relaying environments. Existing works on relay power allocation mainly focus on per transmission power usage, either for the optimal power allocation with a given power budget for certain network performance maximization, or for the minimum level of power consumption per transmission to guarantee some system performance [1]-[4]. These solutions are focused on optimal power allocation without energy limitation. However, when the relays have limited energy, the above studies do not consider the network lifetime.

Existing studies on lifetime maximization via relaying focus on single source-destination (S-D) pair. The problem of relay placement and power assignment for DF cooperative relaying was studied in a multi-node network to maximize the minimum node lifetime [5]. The power allocation there was static as only channel statistics were used. Relay selection and power allocation schemes were devised to prolong the lifetime of an AF relay network with single S-D pair in [6], where a finite number of power levels was assumed. The problem of optimal power allocation to maximize the network lifetime in $\mathrm{AF}$ relay networks was studied in [7] for both single and multiple $\mathrm{S}-\mathrm{D}$ pairs. Instead of relay selection, all relays participate in the data forwarding in that study. Although prolonging network lifetime has been well studied for sensor networks [8], some main differences between lifetime maximization in relay cooperation and the sensor networks (e.g., lifetime definition, link characteristic assumptions, and performance metric) make the results obtained there not applicable to this problem. In addition, maintaining multiple S-D transmissions poses different network setup and requirement from those in sensor networks. To the best of our knowledge, the problem of relay selection for network lifetime maximization in the presence of multiple S-D pairs has not been investigated before.

In this paper, we consider a dual-hop AF relay network consisting of multiple S-D pairs and multiple relays. We define the network lifetime as the time interval over which successful transmission of all S-D pairs can be maintained above the given data rate requirements. Without any knowledge of future channel states, we design relay selection algorithms to maximize the perceived network lifetime at the current time. The perceived network lifetime maximization is shown to be a max-min optimization problem for which the optimal mapping strategy of relays to S-D pairs is sought. We propose a priority search algorithm which provides the optimal solution with linear complexity in the number of relays, as opposed to exponential complexity in the exhaustive search. Furthermore, we propose a suboptimal user-priority-based selection strategy, i.e., the "worst-case" greedy algorithm, with complexity linear in the number of relays and quadratic in the number of S-D pairs. Simulation results show that the performance loss of the "worst-case" greedy algorithm is negligible as compared to the optimal relay selection solution.

\section{NETWORK MODEL}

Consider a dual-hop AF relaying network with $M$ S-D pairs and $N$ relays with $N \geq M$. Each source sends data to its corresponding destination via a relay selected by this pair. Each relay can at most be selected by only one S-D pair. Due to half-duplex transmission, two phases are used for relaying, i.e., from source to relay, and then from relay to destination. We assume orthogonal channels are used over different S-D pairs, and therefore there is no interference among S-D pairs during relaying. The direct link between S-D pair is assumed to exist. 
We assume each source has transmit power $P_{s}$. For the $l$ th $\mathrm{S}-\mathrm{D}$ pair, the channel gains at time $t$ between source and destination, source and the $k$ th relay, and the $k$ th relay and destination are denoted by $h_{0}^{l}(t), h_{1 k}^{l}(t)$, and $h_{2 k}^{l}(t)$, respectively. The transmit power used at the relay for the $l$ th $S$ D pair is $P_{k}^{l}(t)$. The receiver noise is additive white Gaussian noise. Without loss of generality, we assume the noise variance is the same for all links and denote it as $\sigma_{n}^{2}$. With slight abuse of notation, we use time $t$ for both the first and second phase transmissions, to indicate that it is part of the transmission of the same source data. Nonetheless, such notation should not cause confusion, as it essentially indicates the time of a complete dual-hop relay transmission. The received SNR from the $k$ th relay is given by

$$
\gamma_{k}^{l}\left(P_{k}^{l}(t)\right)=\frac{P_{s} P_{k}^{l}(t) b_{k}^{l}(t) c_{k}^{l}(t)}{1+P_{s} b_{k}^{l}(t)+P_{k}^{l}(t) c_{k}^{l}(t)}
$$

where $b_{k}^{l}(t)=\left|h_{1 k}^{l}(t)\right|^{2} / \sigma_{n}^{2}$ and $c_{k}^{l}(t)=\left|h_{2 k}^{l}(t)\right|^{2} / \sigma_{n}^{2}$ are the nominal received signal-to-noise ratio (SNR) with unit transmit power at relay $k$ and the destination (from relay $k$ ), respectively. At the destination, the maximum ratio combining (MRC) technique can be used to add coherently the received signal from the relay path and the direct path, leading to a combined SNR of the two paths. Therefore, the effective endto-end data rate for the $k$ th S-D pair at time $t$ is given by

$$
C_{k}^{l}(t)=\frac{1}{2} \log \left[1+P_{s} a^{l}(t)+\gamma_{k}^{l}\left(P_{k}^{l}(t)\right)\right]
$$

where $a^{l}(t)=\left|h_{0}^{l}(t)\right|^{2} / \sigma_{n}^{2}$ is the nominal received SNR from the direct path, and $1 / 2$ is the bandwidth efficiency factor, reflecting the two-phase transmission. We assume only the current channel state information (CSI) is known for relay selection.

\section{Energy-Aware Relay SELECTION}

\section{A. Definition of Network Lifetime}

We assume that relays are battery powered. Let $\mathcal{E}_{k}(t)$ be the residual energy of relay $k$ at time $t$. The initial energy is then given by $\mathcal{E}_{k}(0)$ for relay $k$. A relay gradually depletes its energy as it participates in forwarding the source message. A relay can no longer cooperate if its required energy for the current transmission is more than its residual energy. To define the lifetime of such a relay network, we use a more direct definition to capture the functionality of the network. That is to maintain the data rate requirement of the end-toend data transmission subject to a limited energy budget at each relay. To define the network lifetime $(L T)$ for multiple S-D transmissions, we first describe per S-D relay transmission lifetime. Let $k_{l}(t)$ be the selected relay for the $l$ th S-D pair at time $t$, and $C_{k_{l}}^{l}(t)$ is the rate achieved at time $t$ with the selected relay $k_{l}(t)$ at $t$. The lifetime for the $l$ th $S-D$ pair assisted by relaying under the selection policy $\pi$ is the time interval during which the end-to-end data rate for this pair is maintained above a minimum required rate $R_{l}$ [7],

$$
L T^{l}=\max \left\{t: C_{k_{l}}^{l}\left(t^{\prime}\right) \geq R_{l}, t^{\prime} \in[0, t] \mid \pi\right\}
$$

for $l=1, \cdots, M$. For multiple S-D pairs, under a given relay selection policy $\pi$, the network lifetime is defined as the time interval within which all the S-D pairs can meet their own target data rate requirement through their respective selected relays. A relay selection policy $\pi$ determines the selected relay $k$ for the $l$ th S-D pair at each time slot $t$. Under this selection policy, the network lifetime is given by

$$
\begin{aligned}
L T & =\max \left\{t: C_{k_{l}}^{l}\left(t^{\prime}\right) \geq R_{l}, \forall l, t^{\prime} \in[0, t] \mid \pi\right\} \\
& =\min _{l}\left\{L T^{l} \mid \pi\right\} .
\end{aligned}
$$

From the above definition, the network lifetime is determined by the S-D pair with the minimum lifetime among all pairs. Furthermore, it is a function of the relay selection policy $\pi$.

Note that the network lifetime definition in (3) is applicable for the case $N \geq M$ assumed in our network model. For the case $N<M$, only a subset of S-D pairs can be assisted by relays, the lifetime definition would need to be modified. It can be shown that with some modification, our proposed relay selection policies in Section IV can still be carried out. However, we will focus on the case $N \geq M$ in this paper.

\section{B. Perceived Network Lifetime and Myopic Policy}

We aim to design a relay selection policy to prolong the network lifetime in the multiuser relaying network. Since the current relay selection affect the future action and thus the network lifetime, without certain knowledge of the future channel state information (CSI) (probabilistically or deterministically), the global optimal selection policy cannot be obtained. Instead, with only the current CSI available, we design the selection policy to maximize the perceived network lifetime at the current time $t$.

The perceived lifetime is the estimated time interval that remains based on the current CSI and residual energy. It has been used in [7] for single S-D pair lifetime maximization. It is computed assuming that the current CSI is unchanged in the future. Let $n_{k}^{l}(t)$ be the perceived lifetime for the $l$ th S-D pair with relay $k$. In each time slot, the perceived lifetime $n_{k}^{l}(t)$ that the $k$ th relay can provide to the $l$ th user is given by [7]

$$
\begin{aligned}
& n_{k}^{l}(t)= \\
& \max \left\{n: P_{s} a^{l}(t)+\frac{\mathcal{E}_{k}(t) P_{s} b_{k}^{l}(t) c_{k}^{l}(t)}{n\left(1+P_{s} b_{k}^{l}(t)\right)+\mathcal{E}_{k}(t) c_{k}^{l}(t)} \geq \gamma_{t h}^{l}\right\}
\end{aligned}
$$

for $l=1, \cdots, M ; k=1, \cdots, N$, and $\gamma_{t h}^{l}$ is the corresponding SNR for the given data rate constraint. The power allocation at the selected relay at the current time $t$ is thus given by

$$
P_{k}(t)=\mathcal{E}_{k}(t) / n_{k}^{l}(t)
$$

for $l=1, \cdots, M$. Based on the perceived lifetime definition for a single S-D pair, the perceived network lifetime in the presence of multiple S-D pairs, at the current time slot $t$, is then given as

$$
n(t ; \pi)=\min _{l}\left\{n_{k_{l}}^{l}(t) \mid \pi\right\}
$$

We focus on myopic relay selection policies which are essentially mapping strategies from S-D pairs to relays at every time 
slot. The optimal mapping strategy is such that the perceived network lifetime at the current time slot is maximized.

Note that if only the perceived network lifetime is concerned, the mapping strategy may not be unique. Indeed, if we fix the S-D pair and relay that gives the worst perceived lifetime, i.e., the perceived network lifetime, there are multiple possibilities of relay selection for the rest S-D pairs. We need to find a unique strategy such that it maximizes the perceived network lifetime in a recursive fashion. That is, the optimal selection strategy should satisfy the condition in following repeated procedure.

P1). The perceived network lifetime at current time $t$, for the set of S-D pairs and relays under consideration, is maximized under $\pi^{*}$

$$
n\left(t ; \pi^{*}\right)=\max _{\pi} n(t ; \pi) .
$$

P2). Among those $\pi$ 's satisfying (8), remove the S-D pair and the selected relay that gives the perceived network lifetime, if the updated set of remaining S-D pairs is not empty, go to P1).

\section{Priority-Based Relay Selection Policies}

In this section, we will provide both optimal and suboptimal relay selection solutions with different complexity. The proposed policies are based on certain priority of the S-D pairs in selecting the relay.

Since the selection policy is only based on the current time $t$, with notation simplicity, in the following description, we drop the time index $t$, with the understanding that the notations are with respect to the time slot $t$.

\section{A. Optimal Solution - Max-Min Optimization}

1) Exhaustive Search: According to the design criterion (8) in the recursive procedure P1)-P2), it is essentially to find the optimal mapping of $M$ S-D pairs to the $M$ out of $N$ relays. One can exhaustively list all the possible relay assignments to the $M$ S-D pairs to build a complete mapping space $S_{\text {exh }}$ consisting all possible mapping strategies, and select the one that determines the maximum of minimum pair-wise perceived lifetime. The total number of possible mapping strategies to be considered is $\frac{N !}{(N-M) !}$.

2) Priority Search Algorithm: The exhaustive search will incur high computation complexity when $M$ and $N$ are large. To obtain the optimal selection solution, we propose a priority search algorithm, where we conduct a search based on the priority of S-D pairs.

For $M$ S-D pairs, there are $M$ ! possible arrangements of priority order. Let $\mathbf{a}^{i}$ be the $i$ th such priority-order vector (PV), where $\mathbf{a}^{i}=\left[a_{1}^{i}, \cdots, a_{M}^{i}\right]^{T}$, and $a_{j}^{i}$ denotes the index of the SD pair with the $j$ th priority. Let $\mathcal{S}_{\mathrm{a}}$ be the complete set of $\mathbf{a}^{i}$ 's: $\mathcal{S}_{\mathbf{a}}=\left\{\mathbf{a}^{1}, \cdots, \mathbf{a}^{M !}\right\}$, For each PV $\mathbf{a}^{i}$, S-D pairs will conduct the relay selection sequentially based on the priority order in $\mathbf{a}^{i}$. The relay selection criterion is to maximize the perceived lifetime of this S-D pair among the relays that have not yet been selected. Once the best relay is selected, it is removed from the set of available relays. After all S-D pairs have made the relay selection, we obtain $\mathbf{k}^{i}=\left[k_{1}^{i}, \cdots, k_{M}^{i}\right]^{T}$, where $k_{j}^{i}$ denotes the selected relay for the $j$ th priority S-D pair under the PV $\mathbf{a}^{i}$, and the corresponding vector of perceived lifetime of all S-D pairs is denote as $\mathbf{n}_{\mathbf{k}}^{i}=\left\{n_{k_{1}^{i}}^{1}, \cdots, n_{k_{M}^{i}}^{M}\right\}$. After obtaining the pair $\left\{\mathbf{k}^{i}, \mathbf{n}_{\mathbf{k}^{i}}^{i}\right\}$ for all $\mathbf{a}^{i}$ 's in $\mathcal{S}_{\mathbf{a}}$, we choose the optimal PV index $i^{o}$, and the mapping strategy $\mathbf{k}^{i o}$ such that the minimum value in $\mathbf{n}_{\mathbf{k}^{i}}^{i}$ is maximized

$$
i^{o}=\arg \max _{i: \mathbf{a}^{i} \in \mathcal{S}_{\mathbf{a}}} \min \left\{n_{k_{l}^{i}}^{l}: n_{k_{l}^{i}}^{l} \in \mathbf{n}_{\mathbf{k}}^{i}\right\}
$$

If such $i^{o}$ is not unique, the set $\mathcal{S}_{\mathbf{a}}$ of $\mathbf{a}^{i}$,s is updated, by only keeping those $\mathbf{a}^{i}$ 's with indices $i$ 's that satisfy (9). After the update, the perceived network lifetimes under $\mathbf{k}^{i}$ for $\mathbf{a}^{i} \in \mathcal{S}_{\mathbf{a}}$ are the same. To determine the mapping strategy $\mathbf{k}^{i}$, we will exclude the S-D pairs leading to the worst perceived lifetime, and decide how to best select the relays for the rest S-D pairs based on the max-min lifetime criterion. In other words, (9) is repeated with the updated $\mathcal{S}_{\text {a }}$ until only a unique $i^{\circ}$ remains. Then we obtain the final mapping strategy for relay selection $\mathbf{k}^{i o}$, and allocate the power for each selected relay according to (6).

The Priority Search Algorithm is summarized in Algorithm 1 . The optimality of the solution obtained by the Priority Search Algorithm is described in the following proposition.

Proposition 1: The solution obtained by the Priority Search Algorithm is identical to that obtained in the exhaustive search.

Proof: We give a brief outline of proof due to the page limitation. Let $\mathcal{S}_{P S}$ be the set of mapping strategies in the priority search algorithm. We have $\left|\mathcal{S}_{P S}\right|=M$ !. Thus it is a subset of $\mathcal{S}_{\text {exh }}$ under the exhaustive search. We show that for any mapping strategy $\mathbf{k} \in \mathcal{S}_{e x h} \backslash \mathcal{S}_{P S}$, there exists a $\mathbf{k}^{\prime} \in \mathcal{S}_{P S}$, such that $\min \left\{n_{k^{\prime}}^{l}: n_{k^{j}}^{l} \in \mathbf{n}_{\mathbf{k}^{\prime}}\right\} \geq \min \left\{n_{k}^{l}: n_{k}^{l} \in \mathbf{n}_{\mathbf{k}}\right\}$. Therefore, the local optimal mapping strategy obtained with in $\mathcal{S}_{P S}$ is the global optimal strategy for $\mathcal{S}_{\text {exh }}$.

\section{B. Suboptimal Solution - "Worst-Case" Greedy Approach}

The Priority Search approach is carried out by exhausting all the possible priority orders of S-D pairs for relay selection. The computational complexity is still relatively high. This motivates us to find a suboptimal approach with low complexity.

In this section, we propose a "worst-case" greedy algorithm, where the priority order of S-D pairs is determined in one-shot, and is used to conduct relay selection. In this algorithm, the highest priority will be given to the S-D pair whose perceived lifetime under the worst relay selection is the shortest. Let $\mathcal{S}_{u}$ and $\mathcal{S}_{r}$ be the set of S-D pairs and relays that are currently under consideration. The current highest priority S-D pair is selected by

$$
l_{*}=\arg \min _{l \in \mathcal{S}_{u}}\left\{\min _{k \in \mathcal{S}_{r}}\left\{n_{k}^{l}\right\}\right\} .
$$

This top priority pair then selects the relay that leads to the longest perceived lifetime

$$
k_{l *}=\arg \max _{k \in \mathcal{S}_{r}}\left\{n_{k}^{l_{*}}\right\} .
$$




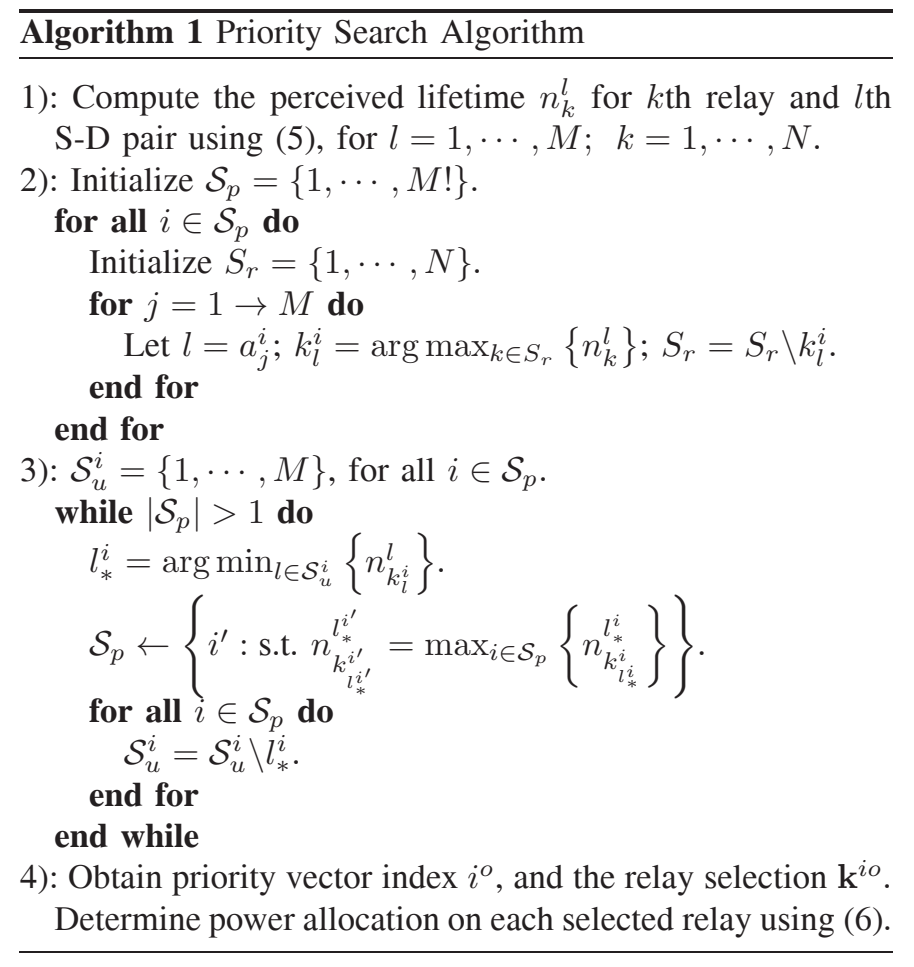

This $l_{*}$ th S-D pair and the selected relay $k_{l *}$ are then removed from $\mathcal{S}_{p}$ and $\mathcal{S}_{r}$, respectively. The above procedure repeats until all the S-D pairs have made their relay selection. The "worst-case" greedy algorithm is given in Algorithm 2.

In this algorithm, we use the "worst-case" criterion to determine the priority order of S-D pairs for selecting a relay. This particular priority order is one of all possible priority orders used in the priority search algorithm, and may not be the optimal one. Thus, the performance is suboptimal. Since only a single priority order is computed and used for relay selection, the complexity of the "worst-case" greedy algorithm is significantly lower than that of the priority search algorithm.

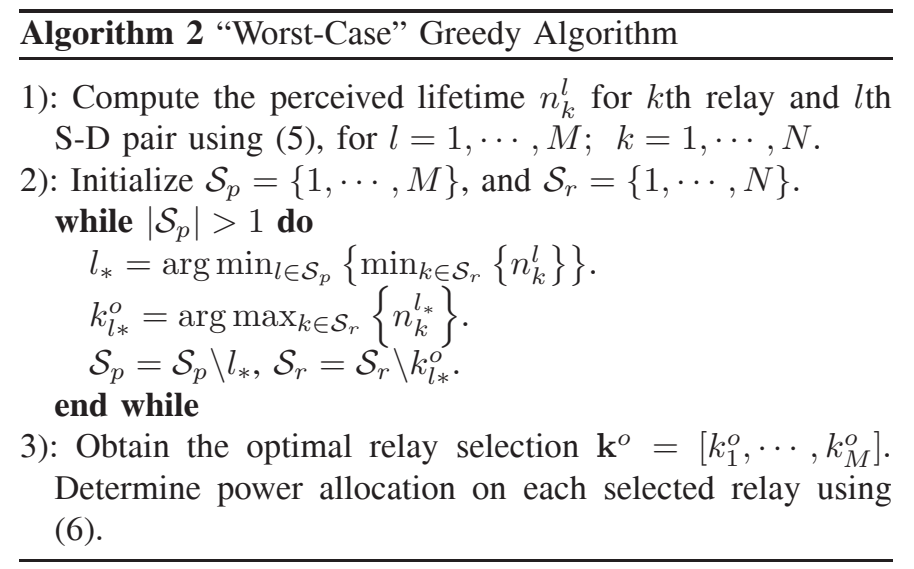

\section{Complexity Comparison}

In this section, we analyze and compare the computational complexity of the proposed relay selection algorithms.
TABLE I

Computational Complexity of RELAy SELECTION

\begin{tabular}{|c|c|}
\hline Algorithm & Complexity \\
\hline Exhaustive search & $\mathcal{O}\left(M N^{M}\right) \sim \mathcal{O}\left(M^{2} N^{M}\right)$ \\
\hline Priority search & $\mathcal{O}\left(N M^{M+1}\right)$ \\
\hline "Worst-case" greedy & $\mathcal{O}\left(N M^{2}\right)$ \\
\hline
\end{tabular}

1) Exhaustive Search: In the exhaustive search, there are total $\frac{N !}{(N-M) !}$ possible mapping strategies for relay selection. Based on the procedure P1)-P2), there may be multiple mapping strategies which provide the best minimum per S-D pair perceived lifetime. In this case, we need to repeat P1)-P2) and the comparison among these strategies until a unique mapping strategy can be determined. Thus, the complexity can be varied between $\mathcal{O}\left(M N^{M}\right)$ and $\mathcal{O}\left(M^{2} N^{M}\right)$ in searching for the unique mapping strategy.

2) Priority Search Algorithm: In the priority search, there are total $M$ ! priority orders to consider. For each priority order, the best relay is selected in turn based on the priority index. The optimal priority order is then chosen based on Algorithm 1. It can be shown that the complexity of the priority search algorithm is $\mathcal{O}\left(N M^{M+1}\right)$, which is linear in the number of relays $N$.

3) "Worst-Case" Greedy Algorithm: In the greedy approach, the top-priority S-D pair selection and the corresponding relay selection procedures are repeated $M$ times until all S-D pairs have selected their relays. It can be shown that the complexity of the "worst-case" greedy algorithm is $\mathcal{O}\left(N M^{2}\right)$, thus the complexity is reduced to linear in the number of relays $N$ and quadratic in the number of S-D pairs $M$.

The complexity comparison among all the algorithms is given in Table I.

4) Trade-off Analysis: We now compare the complexity under the exhaustive search and priority search algorithm. Let $\rho=\frac{M}{N}$ be the network "load" coefficient, which ranges from 0 to 1 . The network is viewed as "light-load" if the number of S-D pairs is significantly less than that of available relays, i.e., $\rho \leq \frac{1}{2}$, "half-load" if $\rho=\frac{1}{2}$, and "heavy-load" if $\frac{1}{2}<\rho \leq 1$. For the "full-load" case $(\rho=1)$, the complexity in the exhaustive search is $\mathcal{O}\left(M^{M+1}\right) \sim \mathcal{O}\left(M^{M+2}\right)$, and that in the priority search is $\mathcal{O}\left(M^{M+2}\right)$, which is equal to that of the upper bound of the exhaustive search. Therefore, in the "full-load" case, the exhaustive search is preferable. When $\rho<1$, the upper and lower bounds of the exhaustive search become $\mathcal{O}\left(M^{2} N^{M}\right)=\mathcal{O}\left((1 / \rho)^{M} M^{M+2}\right)$, and $\mathcal{O}\left(M N^{M}\right)=\mathcal{O}\left((1 / \rho)^{M} M^{M+1}\right)$, while the complexity for the priority search turns out to be $\mathcal{O}\left(N M^{M+1}\right)=$ $\mathcal{O}\left(\frac{1}{\rho} M^{M+2}\right)$. Clearly, the priority search in this case results in much lower complexity. Therefore, in the case $M \ll N$, the relay selection complexity under the priority search algorithm is greatly reduced. Such scenario arises in ad hoc or mesh network applications, when there are many relays available to forward data for a few S-D pairs. 


\section{Simulation Results}

We set the S-D pairs and the relays to be randomly located. The initial energies for all sources are equal to $10 \mathrm{~J}$. We assume fading channels with the path-loss exponent is 2 and the noise variance $\sigma_{n}=10^{-4} \mathrm{~W}$. The correlation of slow fading channel across time slots is 0.99 . We use priority round-robin as the bench mark comparison, where each S-D pair in turn to have the highest priority to select a relay.

Fig. 1 shows the simulated network lifetime vs. data rate under each relay selection algorithm for $M \times N=2 \times 4$, and $4 \times 4$. We see that the performance of the max-min optimal solution and the "worst-case" greedy algorithm are comparable, with the latter only results in a small performance loss. Without considering CSI and residual energy, the user priority round-robin is much worse than the other approaches.

We also plot the lifetime experienced by each S-D pair at different data rate in Fig. 2. As we expected, the optimal solution under the priority search shows gives relatively uniform lifetime experienced by each S-D pair. The "worst-case" greedy approach provides very similar relative lifetime performance across S-D pairs. The priority round-robin approach has the most significant difference among different S-D pairs, as it determines the priority independent of fading channel conditions and residual energy at each relay.

Lastly, to show the efficiency of using different relays, we compare the residual energy of each of the four relays used in Fig. 2 after network lifetime is reached, under target rates $(1.0,1.5,2.0)$ bits/s. Shown Fig. 3, we see that the priority search has the minimum relay residual energy, with efficient use of relays. The "worst-case" greedy approach provides slightly worse residual energy profile, and the priority roundrobin approach leads to largest waste of residual energy.

\section{CONCLUSION}

We have considered the problem of optimally selecting relays for the multiple S-D pairs to maximize the network lifetime. The relay selection strategies are designed to maximize the perceived network lifetime at current time. Viewing the problem as a max-min optimization problem, we have proposed a priority search algorithm to find the optimal solution with reduced complexity in the number of relays. To further reduce the complexity, we have proposed a suboptimal priority-based selection strategy, the "worst-case" greedy algorithm, with complexity linear in the number of relays and quadratic in the number of S-D pairs. Simulation results have demonstrated a negligible performance loss of the "worstcase" greedy algorithm as compared to the optimal relay selection solution.

\section{REFERENCES}

[1] A. Bletsas, A. Khisti, D. P. Reed, and A. Lippman, "A simple cooperative diversity method based on network path selection," IEEE J. Select. Areas Commun., vol. 24, pp. 659-672, Mar. 2006.

[2] Y. Zhao, R. Adve, and T. J. Lim, "Improving amplify-and-forward relay networks: Optimal power allocation vs. selection," IEEE Trans. on Wireless Comm., pp. 3114-3123, Aug. 2007.

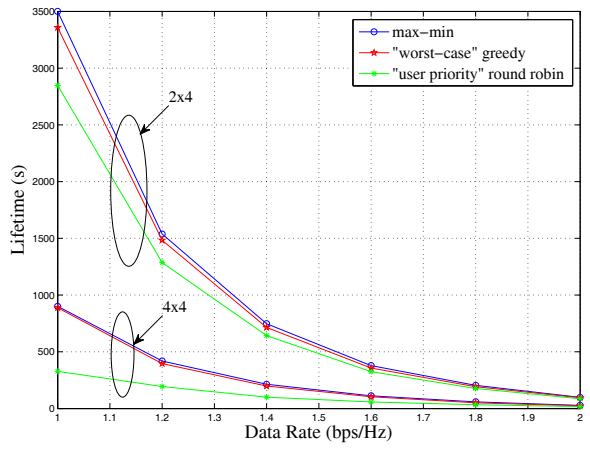

Fig. 1. Network lifetime vs. target data rate $(\mathrm{M}=2$ or $4, \mathrm{~N}=4)$ with $\alpha=0.99$.
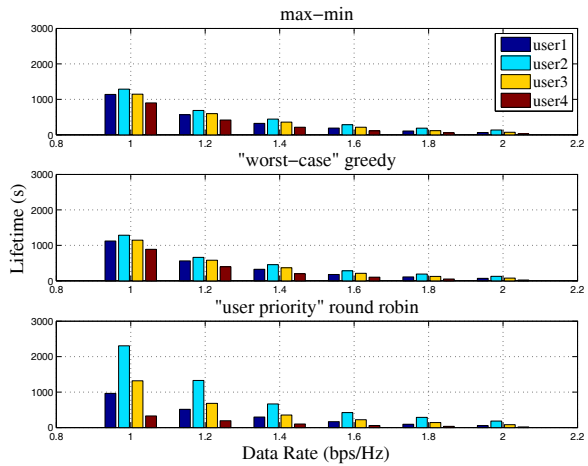

Fig. 2. Lifetime of $\mathrm{S}-\mathrm{D}$ pairs vs. target data rate $(\mathrm{M}=\mathrm{N}=4) .(\alpha=0.99)$

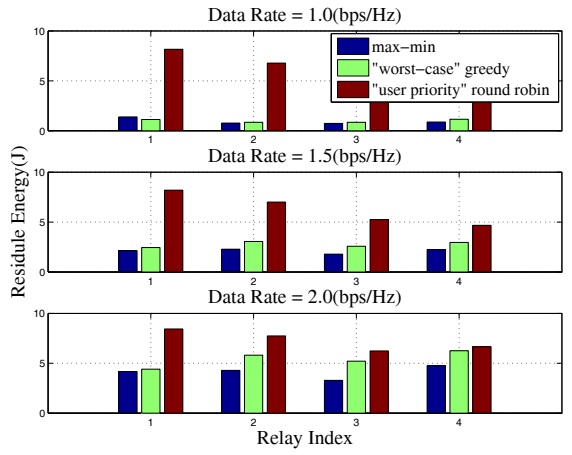

Fig. 3. Relays' residual energy $(\mathrm{M}=\mathrm{N}=4) .(\alpha=0.99)$

[3] M. O. Hasna and M. S. Alouini, "Optimal power allocation for relayed transmissions over Rayleigh-fading channels," IEEE Transactions on Wireless Communications, vol. 3, no. 6, pp. 1999-2004, Nov. 2004.

[4] M. Hajiaghayi, M. Dong, and B. Liang, "Using limited feedback in power allocation design for a two-hop relay OFDM system," in Proc. IEEE Int. Conf. Communications (ICC), vol. 2, Jun. 2009, pp. 1167-1171.

[5] T. Himsoon, W. P. Siriwongpairat, Z. Han, and K. J. R. Liu, "Lifetime maximization via cooperative nodes and relay deployment in wireless networks," IEEE J. Select. Areas Commun., vol. 25, no. 2, pp. 306-317, Feb. 2007.

[6] W.-J. Huang, Y. W. P. Hong, and C. C. J. Kuo, "Lifetime maximization for amplify-and-forward cooperative networks," IEEE Trans. Wireless Commun., vol. 7, pp. 1800-1805, May 2008.

[7] M. Hajiaghayi, M. Dong, and B. Liang, "Maximizing lifetime in relay cooperation through energy-aware power allocation," IEEE Trans. Signal Processing, vol. 58, pp. 4354-4366, Aug. 2010.

[8] J.-H. Chang and L. Tassiulas, "Maximum lifetime routing in wireless sensor networks," IEEE/ACM Trans. Netw., vol. 12, pp. 609-619, 2004. 